\title{
The Total Energy and Momentum Stored in a Particle
}

\author{
Eyal Brodet ${ }^{1}$ \\ ${ }^{1}$ Michaeslon St, Jerusalem, 93707, Israel \\ Correspondence: Eyal Brodet, Michaeslon St, Jerusalem, 93707, Israel. E-mail: eyalbrodet@hotmail.com \\ Received: March 1, 2017 \\ Accepted: March 14, $2017 \quad$ Online Published: March 17, 2017 \\ doi:10.5539/apr.v9n2p65 \\ URL: https://doi.org/10.5539/apr.v9n2p65
}

\begin{abstract}
In this paper we reconsider the conventional expressions given by special relativity to the energy and momentum of a particle. In the current framework, the particle's energy and momentum are computed using the particle's

rest mass, $\mathrm{M}$ and rest mass time, $t_{m}=\frac{h}{M \cdot c^{2}}$ where $t_{m}$ has the same time unit as conventionally used for the light velocity c. Therefore it is currently assumed that this definition of time describes the total kinetic and mass energy of a particle as given by special relativity. In this paper we will reexamine the above assumption and suggest describing the particle's energy as a function of its own particular decay time and not with respect to its rest mass time unit. Moreover we will argue that this rest mass time unit currently used is in fact the minimum time unit defined for a particle and that the particle may have more energy stored with in it. Experimental ways to search for this extra energy stored in particles such as electrons and photons are presented.
\end{abstract}

Keywords: Energy, momentum, special relativity, decay time

\section{Introduction}

In this paper we reconsider the conventional expressions given by special relativity to the energy and momentum of a particle. In the current framework, the energy and momentum are defined with respect to an arbitrary unit of time given in seconds, and it is assumed that this definition describes the total kinetic and mass energy of a particle as given by special relativity. As will be discussed, this arbitrary time unit is related to the particle's rest mass and may correspond to the shortest decay time the particle may have. However in many cases the particle is associated with a larger internal time unit (in the case of stable particles) or a larger decay time (in the case of unstable particles). In this note we suggest to relate the particle's energy to its own internal time unit, which may mean that a particle may have more energy stored within it then conventionally assumed and observed.

This paper is divided into 4 sections. Section 2 describes the theoretical background. Section 3 describes the experimental search for the extra energy suggested in this note and section 4 contains the conclusions.

\section{Theoretical Background}

Using the fact that the universe expands at some velocity, $V_{\exp }$ (Moskowitz, 2016) and that the maximum velocity is the speed of light $\mathrm{c}$, one may define the absolute position of particle $\mathrm{i}$ in within the universe by:

$$
X_{i}=V_{T} \cdot t_{i}
$$

Where $V_{T}$ depends on the particle's velocity with respect to $V_{\exp }$, the speed of light c and the possible hidden variable in time, $f_{r(i)}$ (Brodet, 2010), as will be discussed in more details later on, and $t_{i}$ is an internal time unit of the particle which may be defined by the particle's possible hidden variable in time, $f_{r(i)}$ (Brodet, 2010) such:

$$
t_{i}=\tau \cdot \ln \left(\frac{M}{f_{r(i)}}\right)
$$

From using the fact that any length $X_{i}$ generally corresponds to a complex number, $z=X_{i}^{\prime}+i c^{\prime} t_{i}^{\prime}$, of modulus $X_{i}$ given by:

$$
X_{i}=|\mathrm{z}|=\sqrt{X_{i}^{\prime 2}+c^{\prime 2} \cdot t_{i}^{\prime 2}}
$$


Where $t_{i}$ may be the same particle's internal time as in equation $1, c^{\prime}$ is some velocity and $X_{i}^{\prime}$ is some length.

We may deduce a relationship between the 3 dimensional of space $\left(X_{i}^{\prime}\right)$, the dimension of time $\left(t_{i}\right)$ and the combined dimension of space-time $\left(X_{i}\right)$.

This could be modified in order to correspond to special relativity, where $X_{i}$ may be a related to the length of a 4-vector $\left(\frac{X_{i}^{\prime}}{\sqrt{2}}, \frac{c \cdot t_{i}}{\sqrt{2}}\right)$ such:

$$
X_{i}=\sqrt{\frac{X_{i}^{\prime 2}}{2}+\frac{X_{i}^{\prime 2}}{2}-\frac{c^{2} \cdot t_{i}^{2}}{2}}
$$

Where $t_{i}$ is the same particle's internal time as in equation $1, X_{i}^{\prime}$ may be some arbitrary position in 3 dimensions related to particle $\mathrm{i}$ in its own rest frame. In this paper we will take $X_{i}^{\prime}=0$ and assume $c \cdot t_{i}$ as particle's $\mathrm{i}$ invariant position in any moving frame observed by any object, $\mathrm{c}$ is the speed of light and $X_{i}^{\prime \prime}$ is the position of particle $\mathrm{i}$ with respect to the expanding universe which may be given by: $X_{i}^{\prime \prime}=V_{r(T)} \cdot t_{i}$. The definition of $V_{r(T)}$ will be given later on in this paper:

Therefore equation 3, which describes the absolute position of particle $\mathrm{i}$ in the universe may be given in terms of $V_{T}$ and $V_{r(T)}$ such:

$$
V_{T} \cdot t_{i}=\sqrt{\frac{\left(V_{r(T)} \cdot t_{i}\right)^{2}}{2}-\frac{c^{2} \cdot t_{i}^{2}}{2}}
$$

In the light of the possibility of $f_{r(i)}$, the term involving $c \cdot t_{i}$ can be viewed as involving the internal information of particle $\mathrm{i}$. Therefore the value of $\mathrm{c}$ may be viewed as only a part of particle's i velocity which may also depend on $f_{r(i)}$. Therefore, we may change $\mathrm{c}$ into $c_{i}$ such: $c_{i}=\frac{\lambda_{i}}{t_{i}}$ where $c \neq c_{i}$ and $\lambda_{i}$ is the relevant size of particle i. The above may give equation 3 and 3 a in terms of $f_{r(i)}$ such:

$$
X_{i}=\sqrt{\frac{X_{i}^{\prime \prime 2}}{2}-\left(\frac{\lambda_{i}}{2 \cdot \tau \ln \left(\frac{M}{f_{r(i)}}\right)}\right)^{2} \cdot t_{i}^{2}}
$$

and equation $3 \mathrm{a}$ by:

$$
V_{T} \cdot t_{i}=\sqrt{\frac{\left(V_{r(T)} \cdot t_{i}\right)^{2}}{2}-\left(\frac{\lambda_{i}}{2 \cdot \tau \ln \left(\frac{M}{f_{r(i)}}\right)}\right)^{2} \cdot t_{i}^{2}}=\sqrt{\frac{\left(V_{r(T)} \cdot t_{i}\right)^{2}}{2}-\frac{\left(c_{i} \cdot t_{i}\right)^{2}}{2}}
$$

One can rearrange equation 4 such:

$$
0=\frac{X_{i}^{\prime 2}}{2}-X_{i}^{2}-\left(\frac{\lambda_{i}}{2 \cdot \tau \ln \left(\frac{M}{f_{r(i)}}\right)}\right)^{2} \cdot t_{i}^{2}=\frac{X_{i}^{\prime \prime 2}}{2}-X_{i}^{2}-\frac{c_{i}^{2} \cdot t_{i}^{2}}{2}
$$

and define it as some function, $f\left(X_{i}^{\prime \prime}, t_{i}\right)$ such:

$$
f\left(X_{i}^{\prime \prime}, t_{i}\right)=0=\frac{X_{i}^{\prime 2}}{2}-X_{i}^{2}-\frac{\left(c_{i} \cdot t_{i}\right)^{2}}{2}
$$

It would be possible to attempt to derive the possible total energy and momentum from the function $f\left(X_{i}^{\prime \prime}, t_{i}\right)$. The function, $f\left(X_{i}^{\prime \prime}, t_{i}\right)$, is based of course on the basic relation of space and time given by:

$$
X=V \cdot T
$$

Equation 7 was the basis for the theory of special relativity and the derivation of the expressions for the object's energy and momentum. 
It is understood, that the energy and momentum given by special relativity is evaluated with respect to some defined time unit (as will be discussed later), conventionally given in the unit of seconds. Therefore, it is assumed by special relativity that the energy and momentum evaluated for this defined time unit describes the total energy of the particle (excluding its potential energy) and that the energy of a particle does not depend on the measurement time itself. In this note we will reexamine this assumption and will derive different and perhaps more comprehensive definitions for an object's energy and momentum.

Therefore, using the particle's absolute position as defined above and the resulted function $f\left(X_{i}^{\prime \prime}, t_{i}\right)$ which may follow, one may define the energy and momentum of a particle as the derivatives of $f\left(X_{i}^{\prime \prime}, t_{i}\right)$ with respect to $\operatorname{space}\left(X_{i}^{\prime \prime}\right)$ and time $\left(t_{i}\right)$ such:

$$
\frac{\partial}{\partial X_{i}^{\prime \prime}} f\left(X_{i}^{\prime \prime}, t_{i}\right)=X_{i}^{\prime \prime}
$$

and

$$
\frac{\partial}{\partial t_{i}} f\left(X_{i}^{\prime \prime}, t_{i}\right)=-c_{i}^{2} \cdot t_{i}
$$

The derivative with respect to $X_{i}^{\prime \prime}$ may de identified as momentum ,P, and the derivative with respect to time may be identified as energy, E,. For the energy case:

$$
E_{i(\text { part })}=c_{i}^{2} \cdot t_{i}
$$

and for the momentum case:

$$
P_{i(\text { part })}=X_{i}^{\prime \prime}=V_{i}^{\prime \prime} \cdot t_{i}
$$

The definition for energy and momentum given by equations 8 and 9 above means that we have to know what is the time, $t_{i}$, the factor $\lambda_{i}$ and velocity $V_{t}^{\prime \prime}$ of the particle in order to know its energy and momentum. These definitions represent only a part of the particle's total energy and momentum and therefore are still not in the conventional dimensions for energy and momentum as will be described later on. Let us now consider what $t_{i}$ and $V_{t}^{\prime \prime}$ may be for any particle in a range of physical conditions. Let us first consider the particle's velocity, $V_{t}^{\prime \prime}$. From equation 7 we learn the basic relation between velocity and the particle's position and time. Since we may assume that equation 7 is always valid, we may ask ourselves what is the value of $\mathrm{V}$ when the particle is at rest and therefore presumably has $V_{t}^{\prime \prime}=0$ ?. Since we assume that a particle at rest does have some objective position $\mathrm{X}$ with respect to the expanding universe, then we may assume that it has some velocity $V_{t}^{\prime \prime}$. The question is now with respect to what one should define the particle's velocity when it is at rest. One possibility is to define the particle's velocity with respect to the center of the universe, $\vec{V}_{\exp }$. Since the universe expands from its center in all directions, we should use only the absolute value of its velocity, i.e. $V_{\text {exp }}=\left|\vec{V}_{\text {exp }}\right|$. This would be an objective definition that could be defined when the particle is at rest and when it is in motion. The value $V_{\exp }$ may be estimated from (Moskowitz, 2016). The choice of the velocity with respect to the center of the universe is somewhat arbitrary, but it allows a common reference velocity for all particle's in all location in the universe. Therefore, we may define $V_{\text {exp }}$ as the particle's velocity at rest. Moreover we may define the velocity $V_{r}$ as the velocity of the particle with respect to a coordinate system that did not experience any acceleration and is not moving. Consequently the total velocity of the particle with respect to the expanding universe may be given from special relativity velocity addition rule where we add $V_{\exp }$ to the relative particle velocity, $V_{r}$ giving the particle a total relative velocity of $V_{r(T)}$ which is said to be equal to $V_{t}^{\prime \prime}$ such: $V_{r(T)}=V_{t}^{\prime \prime}$.

The knowledge of whether a particle or a coordinate system is moving or not(i.e. has $V_{r}=0$ or not) may be evaluated by measuring its electric charge in its own rest frame which is experimentally shown to depend on the particles kinetic energy through the running of the coupling constant, $\alpha_{\text {OFD }}$ (Coupling constant, 2017). In fact the relationship between $\alpha_{O E D}$ and the Fermi constant $G_{F e r m i}$ means that the mean lifetime at the rest frame of particles such as muon's and tau's depend on these particle's velocity. This violates special relativity which considers the particle's decay time at its own rest frame as independent to its velocity. In this paper we attempt to resolve this violation.

Therefore the value $V_{r(T)} \cdot t_{i}$ may give particle's i position with respect to the expanding universe as was discussed in the context of equation 3a. Next we have to understand what is the relevant value of the time $t_{i}$, we have to use in the above equations. Similarly to the velocity case, also here the value of the time, $t_{i}$, has to 
defined in all the known particle physical conditions and should be an objective definition. The natural possibility for the above may be to use the particle's own decay time for unstable particles or the particle's internal time as may be defined from the particle's possible hidden variable in time, $f_{r}$ given by (Brodet, 2010):

$$
f_{r(i)}=M \cdot e^{-\frac{t}{\tau}}
$$

Where $M$ is the particle's mass, $\tau$ is a shape variable determining the shape of the $f_{r}$ distribution and $t_{i}$ is the internal time of particle i.

Equation 10 could be rearranged to give $t_{i}$ of the particle such:

$$
t_{i}=\tau \ln \left(\frac{M}{f_{r(i)}}\right)
$$

From the above, and including the special relativity boost factor $\gamma_{b}$ for the time, we may define the particle's energy as:

$$
E_{\text {total } \left.(i) \_ \text {(part }\right)}=c_{i}^{2} \cdot \gamma_{b} \cdot \tau \ln \left(\frac{M}{f_{r(i)}}\right)
$$

When considering particle $\mathrm{i}$ which is moving at relative velocity $V_{12}$ with respect to another object, its total energy, $E_{\text {total }(i)}$ may be given, including the relativistic time $\gamma_{i} \cdot t_{i}$, by:

$$
E_{\text {total }(i) \_ \text {(part) }}=c_{i}^{2} \cdot \gamma_{i} \cdot t_{i}
$$

Where $\gamma_{i}$ the modified boost factor is first suggested in Brodet (2016):

$$
\gamma_{i}=\frac{1}{\sqrt{1-\frac{V_{12}^{2}}{\left(c+\frac{a 1}{f_{r(i)}}\right)^{2}}}}
$$

In the above equation $V_{12}$ is the relative velocity between coordinate system 1 and 2 and $a 1$ is a constant with yet an unknown value. Note that $V_{12}$ is not necessarily equal to $V_{r(T)}$ since we may have a situation where $V_{r}=0$ and therefore $V_{r(T)}=V_{\text {exp }}$, while $V_{12}$ with respect to some frame may be very different. The meaning of this difference will be discussed later on in this paper. Note that equations 12 and 13, are still not in the conventional energy dimensions, and therefore represent only a part of the particle's total energy as will be described later on.

The boost factor, $\gamma_{i}$ may also be given by:

$$
\gamma_{i}=\frac{1}{\sqrt{1-\frac{V_{12}^{2}}{c_{i}^{2}}}}
$$

For example, let us calculate the muon's rest energy according special relativity and compare it to the total energy of the muon given by equation 13 :

Therefore according special relativity:

$$
E_{s r}=M_{\mu} \cdot c^{2}
$$

Transforming $M_{\mu}$ into seconds using:

gives

$$
t_{m}=\frac{h}{M_{\mu} \cdot c^{2}}
$$




$$
E_{s r}=3.51 * 10^{-6} \mathrm{~m}^{2} / \mathrm{s}
$$

and using equation 13 while substituting the muon's mean lifetime such(since the value of $\gamma_{i}$ is equal 1 , such $\left.\gamma_{i}=1\right)$ :

$$
t_{\text {mean }}=\tau \ln \left(\frac{M}{f_{r(\text { mean })}}\right)=2.196 * 10^{-6} \mathrm{~s}
$$

and taking $c_{i} \approx c$, i.e. to be around the value of the speed of light $c=3 \cdot 10^{8} \mathrm{~m} / \mathrm{s}$ and assuming $\gamma_{i}=1$, we get:

$$
E_{\text {total(mean })}=1.98 \cdot 10^{11} \mathrm{~m}^{2} / \mathrm{s}
$$

Therefore we can see that the total muon's energy at rest given from equation 13 is much larger than the rest energy per unit of seconds related to mass given from special relativity. Consequently one may interpret the conventional energy described by special relativity as the minimum energy of the particle, which is related to the total particle's energy by:

$$
E_{\text {total }(i)}=E_{\min (i)} \cdot \frac{t_{i}}{t_{m(i)}}
$$

Where $E_{\text {total }}$ is given by equation $13, E_{\min (i)}=E_{s r}$ and is the minimum particle's energy, $t_{i}$ is particle's i decay time and $t_{m(i)}$ is the time unit related to rest mass of particle i which is calculated from equation 17 .

If we substitute the $t_{i}=\tau_{\mu}$ and $t_{m(i)}$ calculated from equation 17 we get:

$$
E_{\text {total }}=1.128 \cdot 10^{17} \cdot E_{\text {min }}
$$

Therefore the above suggests that a fraction of the muons may have around $1.128 \cdot 10^{17}$ times more energy at rest than it is known from equation 16.

In fact, according to the suggestion in this paper, the above calculation includes only a part of the particle's energy but it may provide a rough calculation. The total value of the particle's energy at rest also includes the suggested non-zero momentum contribution to the rest energy value, such:

$$
\sqrt{\frac{m_{i}^{2} c_{i}^{4}}{1-\frac{v_{\exp }^{2}}{c_{i}^{2}}}+\frac{m_{i}^{2} v_{\exp }^{2} c_{i}^{2}}{1-\frac{v_{\exp }^{2}}{c_{i}^{2}}}}=m_{i(\exp )} c_{i}^{2}=E_{i_{-} \operatorname{rest}(\exp )}
$$

Where $m_{i(\exp )}=\gamma_{\exp } \sqrt{m_{i}^{2}+\frac{m_{i}^{2} v_{\text {exp }}^{2}}{c_{i}^{2}}}, \gamma_{\text {exp }}=\frac{1}{\sqrt{1-\frac{v_{\text {exp }}^{2}}{c_{i}^{2}}}}$ and $E_{i_{-} r e s t(\exp )}$ is the particle energy at rest including the possible non-zero momentum contribution.

In order for the above to agree with experimental observations the value of constant $a 1$ in the expression for $\gamma_{i}$ given in equation 14 , has to depend on $V_{r(T)}$ and $f_{r(i)}$ such:

$$
\gamma_{i}=\frac{1}{\sqrt{1-\frac{V_{12}^{2}}{\left(c+\frac{a 1\left(V_{r(T)}, f_{r(i)}\right)}{f_{r(i)}}\right)^{2}}}}
$$

Which means that in large values of $V_{r(T)}$ we don't necessarily have more kinetic energy stored in a particle then currently measured and excess of energy may only exist in lower values of $V_{r(T)}$.

Therefore the expression for the total energy of a particle, including the suggested time affect, may be given by: 


$$
E_{\text {total }(i)}=\gamma_{i} \cdot M_{i(\exp )} \cdot c_{i}^{2} \cdot \frac{t_{i}}{t_{m}}
$$

And the expression to total momentum of the particle may be given by:

$$
P_{\text {total }(i)}=\gamma_{i} \cdot V_{r(T)} \cdot M_{i(\exp )} \cdot \frac{t_{i}}{t_{m}}
$$

In the next section we will discuss how one may attempt to reveal and measure the particle's total energy.

The possible reason that this extra energy have not observed so far, may be due to the fact that it was not looked for at relatively low velocities and that conventional energy measurements of a particle takes a very short period of time. This conventional measurement time period may be much shorter than the internal time unit associated to the measured particles as suggested in equation 21, and hence may not measure the total particle's energy. The experimental background behind conventional energy measurement and a possible way to search for extra energy that may be stored within particles will be discussed in the next section.

The expression for $E_{\text {total }(i)}$ given in equation 21 may include also the particle's potential energy which may be included in the particle's decay time as first suggested in Brodet (2013) by:

$$
t_{i}^{\prime}=t_{i}+\frac{c 1}{f_{\text {potential }}}
$$

Where $\mathrm{c} 1$ is a constant and $f_{\text {potential }}$ is related to the particle's potential energy for example in the electric potential case (Brodet, 2012):

$$
f_{\text {potential }}=\left|\frac{k Q_{1} \cdot Q_{2}}{r_{12}}\right|
$$

Therefore particle's i total energy may be expressed by:

$$
E_{\text {total }(i)}^{\prime}=\gamma_{i} \cdot M_{i(\exp )} \cdot c_{i}^{2} \cdot \frac{t_{i}^{\prime}}{t_{m}}
$$

Therefore $E_{\text {total }(i)}^{\prime}$ may describe the total energy of particle $\mathrm{i}$ which is depends on all the currently known energies namely kinetic, mass and potential energies.

\section{Experimental Way to Measure the Particle's Total Energy}

In the case the above suggestion is correct; there should be some experimental evidence for that. When we consider the conventional energy measurement of a particle's energy in standard particle physics detectors such as DELPHI (The DELPHI Collaboration, n.d.), we realize that length of time we attempt to measure the particle's energy using the energy detector, is very short. In order to test the energy expression suggested in equation 21, one may suggest building an energy detector which contains many more scintillator layers which are separated by a much thinner blocking material(for example iron) such that the shower process experienced by an incoming electron or photon, may take much more time. In this way, one may compare the energy measurement of an electron or photon in this experimental setup, to its known initial energy and test equation 21 . Similarly, one may build several energy detectors with a range of iron and scintillator layers thickness and compare the energy measurement obtained for the same initial incoming electron or photon. Thereby comparing any possible differences between the energy measured in each of these several of energy detectors, and attempt to relate it to equation 21. As we do not know initially the $f_{r(i)}$ and $t_{i}$ values of the electrons or photons the suggestion is perform the above energy measurement using many electrons or photons with known conventional initial energy (evaluated from their frequency or velocity), and analyze the result by averaging over large electrons or photons samples of events.

\section{Conclusions}

The conventional energy and momentum description given by special relativity were discussed. It was argued that in the conventional way the energy and momentum are defined with respect to the particle's minimum time unit. It was furthermore discussed that since the particle may be associated with a time unit that is larger than its minimum time unit, the particle may have in fact more energy stored within it than is currently known. 
Experimental ways to search for such extra energy stored within particles were suggested by modifying conventional energy detectors.

\section{References}

Brodet, E. (2010). The possibility of a hidden variable in time. Physics Essays, 23(4).

Brodet, E. (2012). A new look at the transition between potential and kinetic energy. Physics Essays, 25(1).

Brodet, E. (2013). Hypothesized lepton inner discreteness. Physics Essays, 26(2), 247-250.

Brodet, E. (2016). The Relationship Between the Possibility of a Hidden Variable in Time, Possible Photon Mass, Particle's Energy, Momentum and Special Relativity. Applied Physics Research, 8(6), 13.

Coupling constant. (2017). In Wikipedia, the free encyclopedia. Retrieved from https://en.wikipedia.org/wiki/ Coupling_constant

Moskowitz, C. (2016). The expansion speed of the universe. In Space.com. Retrieved August 30, 2016, from http://www.space.com/17884-universe-expansion-speed-hubble-constant.html

The DELPHI Collaboration. (n.d.). The DELPHI detector. Retrieved from http://delphiwww.cern.ch/

\section{Copyrights}

Copyright for this article is retained by the author(s), with first publication rights granted to the journal.

This is an open-access article distributed under the terms and conditions of the Creative Commons Attribution license (http://creativecommons.org/licenses/by/4.0/). 